\title{
Anomalous muscle bellies in the flexor compartment of forearm - A case report
}

\author{
Sunita U Sawant ${ }^{1 *}$, Sunil M Kolekar ${ }^{2}$
}

$\left\{{ }^{1}\right.$ Associate Professor, Department of Anatomy $\left\{{ }^{2}\right.$ Associate Professor, Department of Physiology $\}$

Alluri Sitarama Raju Academy of Medical Sciences, Eluru, Andhra Pradesh, INDIA.

Email: sawantsunita@yahoo.co.in

Abstract Introduction: The muscular variations of upper limb are common. Their importance becomes apparent with regard to surgical approaches for various clinical conditions involving the upper limb. During routine dissection classes to undergraduate medical students, we came across two additional muscle bellies in the flexor compartment of the left forearm of a male cadaver arising from the under surface of flexor digitorum superficialis (FDS) and were found to be inserted into flexor digitorum profundus (FDP) and flexor pollicis longus (FPL) separately. The above two accessory muscle bellies are also called Gantzer's muscles. In another male cadaver, an accessory muscle belly arising from the flexor digitorum superficialis of the right forearm was found to be inserted into the flexor digitorum profundus (FDP). Muscle anomalies of the upper extremity are recognized causes of peripheral nerve disorder. Awareness of these variations is necessary to avoid complications during radio-diagnostic procedures or surgeries in the upper limb.

Keywords: Flexor digitorum profundus, Flexor digitorum superficialis, Flexor pollicis longus, Gantzer's muscle

*Address for Correspondence:

Dr. Sunita U Sawant, Associate professor of Anatomy, ASRAM Medical College, ELURU-534005, West Godavari Dist., Andhra Pradesh, INDIA.

Email: sawantsunita@yahoo.co.in

Received Date: 20/07/2016 Revised Date: 12/09/2016 Accepted Date: 01/10/2016

DOI: https://doi.org/10.26611/1001111

\begin{tabular}{|l|l|}
\hline \multicolumn{2}{|c|}{ Access this article online } \\
\hline Quick Response Code: & \multirow{2}{*}{ Website: } \\
& www.medpulse.in \\
\cline { 2 - 2 } & \\
\hline
\end{tabular}

\section{INTRODUCTION}

Flexor digitorum superficialis (FDS) is a muscle belonging to the superficial muscle group of the forearm. It arises from the common flexor origin over the medial epicondyle of the humerus, from the anterior band of the ulnar collateral ligament of the elbow, from the medial margin of the coronoid process of the ulna and from the anterior oblique line of the radius. At the wrist the tendons arrange themselves in two groups, a superficial one, of the tendons for the middle and ring fingers, and a deep one, of the tendons for the index and little fingers. At the base of the proximal phalanx, each tendon splits into two slips to allow the tendon of flexor digitorum profundus (FDP) to pass through; the slips then rejoin and decussate forming a shallow groove and finally redivide to be inserted into the sides of the middle phalanx ${ }^{1}$. The flexor digitorum superficialis muscle has been grouped as a retrogressive muscle as it represents remnants of connections between two sheets of muscles. On the other hand, the occasional separation [up to their origins] of individual muscle bellies has been considered as a progressive variation in phylogeny ${ }^{2}$. The primary function of FDS is flexion of the middle phalanges of the fingers at the proximal interphalangeal joints. On prolonged contraction, it flexes the metacarpophalangeal joints and wrist joint. Digits of hand perform many fine movements which are essential for our daily life. Therefore a proper anatomical and surgical knowledge of muscular variations is essential to avoid errors in diagnosis and treatment. Variations of forearm flexors have been reported by Anatomists as early as 1813. Gantzer described two accessory muscles in the human forearm which are named Gantzer's muscle. This muscle arises as small belly from forearm flexors and is inserted either into FPL or $\mathrm{FDP}^{3}$. The Gantzer's muscle mostly originated from the medial epicondyle of the humerus or 
from the under surface of FDS muscle. The Gantzer's muscle has clinical importance as it may compress both the median nerve and its branch, anterior interosseous nerve. Proper knowledge of muscular variations is essential not only for anatomists but also for surgeons. Accessory bellies and the tendons of the muscles are surgically noteworthy. Forearm flexors are known to exhibit such variations. Such anomalous muscle bellies should be kept in mind while approaching the forearm for FDS tendon transfer and other surgical procedures around $i t^{4}$.

\section{CASE REPORT}

During routine cadaveric dissection done for the undergraduate students at the department of Anatomy, ASRAM Medical College, Eluru, A.P., India, we observed two accessory muscle bellies in the left upper limb which took their origin from the under surface of FDS muscle and were inserted into flexor digitorum profundus muscle and flexor pollicis longus muscles separately (Fig.1 and Fig.2 ). In another cadaver an accessory muscle belly in the right upper limb was found to be originating from FDS and inserted into flexor digitorum profundus muscle (Fig. 3).

In one cadaver, the Gantzer's muscle to the flexor pollicis longus in the left upper limb was crossing superficial to anterior interosseous nerve and ulnar artery from medial to lateral side (Fig. 2). In Volkman's ischaemic contracture, contraction of this accessory belly (Gantzer's muscle) may lead to entrapment of the anterior interosseous nerve, which may require lengthening/releasing of such accessory belly (Gantzer's muscle) along with the principal belly of flexor pollicis longus. In the same upper limb, another Gantzer's muscle to the FDP was running distally and obliquely crossing the ulnar artery superficially from medial to lateral side (Fig. 2). While median nerve was found passing between the flexor digitorum superficialis and this Gantzer's muscle.

These additional muscle bellies may lead to median nerve compressive neuropathies or vascular compressions. In the another cadaver, Gantzer's muscle in the right upper limb was found running distally and obliquely, crossing the ulnar artery superficially from lateral to medial side and then inserted into the flexor digitorum profundus (Fig. 3). The median nerve was found emerging between the FDS and the Gantzer's muscle.

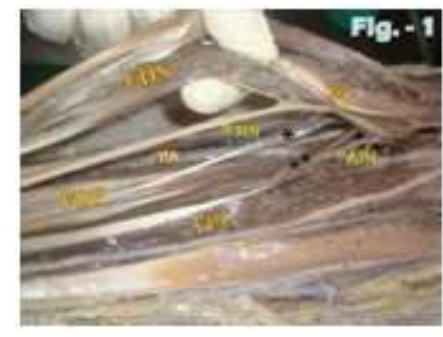

Figure 1: Showing two Gantzer's muscles, the accessory muscle bellies, inserting separately into the flexor digitorum profundus (FDP) and flexor pollicis longus (FPL) in the left upper $\operatorname{limb}$

FDS - Flexor Digitorum Superficialis; PT- Pronator Teres;

* - Gantzer's Muscle inserted to FDP; ** - Gantzer's Muscle inserted to the FPL; MN - Median Nerve; AIN - Anterior Interosseous Nerve; UA - Ulnar Artery.

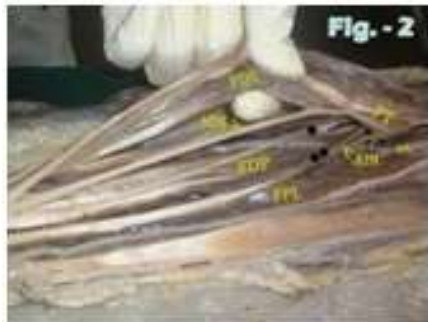

Figure 2: Showing the relations of Gantzer's muscle-bellies to the median nerve, anterior interosseous nerve and ulnar artery in the left upper limb

FDS - Flexor Digitorum Superficialis; FDP - Flexor Digitorum Profundus; FPL - Flexor Pollicis Longus; PT - Pronator Teres; * - Gantzer's Muscle inserted to FDP; ** - Gantzer's Muscle inserted to the FPL; MN - Median Nerve; AIN - Anterior Interosseous Nerve; UA - Ulnar Artery.

\section{DISCUSSION}

The most common singular point of origin of Gantzer's muscle is the under the surface of the FDS, same as in the present case. The occurrence of Gantzer's muscle is lowest in European Caucasians and highest in the Blacks with the Asian Japanese being midways and in Indian population $(46.03 \%)$ it lies between the European Caucasians and the Asian Japanese ${ }^{5,6}$. The presence of additional muscles (as observed in the present case) may be due to the incomplete cleavage of the forearm flexor muscles during development as the deep layer of the flexor muscle mass gives rise to the FDS, FDP and FPL $^{6,7}$. The variant muscle bellies and tendons like this should be kept in mind to avoid clinical complications during radiodiagnostic procedures or surgical approach of these regions; the accessory heads has been implicated in anterior interosseous nerve syndrome ${ }^{8}$. The surgical anatomy of this variation is important for the orthopedic surgeons since this muscular variation can cause chronic inflammation, nerve compressions, tenosynovitis and may 
require surgical excision ${ }^{9}$. Vasavi Rakesh $\mathrm{G}$ et $a l^{4}$ reported the origin of two accessory bellies from the undersurface of the flexor digitorum superficialis muscle inserting into two deep flexors of the forearm (FDP and FPL). Binod Kumar Tamang et $a l^{10}$ observed that the accessory head of flexor pollicis longus was present in $13(43.3 \%)$ cadavers arising from FDS (common origin). Soubhagya R. Nayak et $a l^{6}$ observed the triple gantzer's muscle having common origin from the under cover of the FDS fibers and by fibrous band above the insertion of brachialis and inserting into FDP, FDS and FPL.

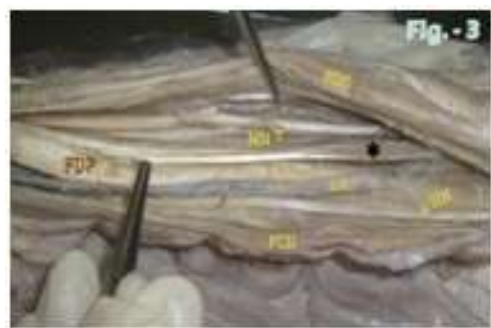

Figure 3: Showing the relations of Gantzer's muscle to the median nerve and ulnar artery in the right upper limb.

FDS - Flexor Digitorum Superficialis; FDP - Flexor Digitorum Profundus; FCU - Flexor Carpi Ulnaris; * - Gantzer's Muscle inserted to FDP; MN - Median Nerve; UN - Ulnar Nerve; UA - Ulnar Artery.

The anatomical variations and abnormalities of the muscles can be detected by imaging techniques, such as computed tomography and magnetic resonance imaging. Identification of variations is important for clinical diognosis and to plan for any surgical procedures. Presence of an accessory muscle may simulate a ganglion or a soft tissue tumour or if in close proximity to a nerve, it may cause pressure neuritis and produce symptoms such as carpal tunnel syndrome, Kiloh-Nevin Syndrome or Pronator Syndrome. FDS has been used as a motor for a wide variety of tendon transfer operations in the hand ${ }^{3}$. We conclude that the knowledge of such variations is very important for radiologists, surgeons, orthopedic surgeons and plastic surgeons. Such anomalous muscle bellies should be kept in mind while approaching the forearm for FDS tendon transfer and other surgical procedures around it.

\section{REFERENCES}

1. Vichare N. A. Anomalous Muscle Belly of the Flexor Digitorum Superficialis. The Journal Of Bone And Joint Surgery 1970; 52, 4, 757-59.

2. Bergman RA, Afifi AK, Miyauchi R. Illustrated encyclopaedia of human anatomic variation: opus I: muscular system: alphabetical listing of muscles: flexor digitorum superficialis, from $<w w w$. anatomyatlasses. org/anatomic variants/muscular system/text/f/17flexor. shtml.> (Retrieved on 7th September, 2006).

3. Takkallapalli Anita1, Sanjay Kalbande, Krishnamurthy Asha, Dattatray Dombe and Neelee Jayasree A Unique Variation of Flexor Digitorum Superficialis Muscle and Its Clinical Significance J Life Sci 2012; 4, 1, 39-43.

4. Vasavi Rakesh G, Bhagath Kumar Potu, Raghu Jetti, Venkata Ramana Vollala, Thejodhar P Rare Origin of Two Accessory Bellies from the Undersurface of the Flexor Digitorum Superficialis Muscle. Eur J Gen Med 2009; 6, 1, 57-59.

5. Pai MM, Nayak SR, Krishnamurthy A, Vadgaonkar R, Prabhu LV, Ranade AV. The accessory heads of flexor pollicis longus and flexor digitorum profundus: Incidence and morphology. Clin Anat 2008; 21, 252-58.

6. Soubhagya R. Nayak, Suranjali Sharma, Hasi Dasgupta and Kalyan Bhattacharya. Presence of triple gantzer's muscle - a rare anatomical variation. NUJHS Vol. 3, No.1, March 2013, ISSN 2249-7110

7. Jones M, Abrahams PH, Sanudo JR, Campillo M. Incidence and morphology of accessory heads of flexor pollicis longus and flexor digitorum profundus (Gantzer's muscles). J Anat 1997; 191, 451-55.

8. Potu BK, Gorantla VR, Pulakunta T, Rao MS, Mamatha T, Vollala VR, Nayak SR. Accessory head of flexor pollicis longus muscle and its significance in anterior interosseous nerve syndrome: case report and review. Int J Morphol. 2007; 25, 911-914.

9. Vishal KUMAR, Naveen NS, Murlimanju BV, Prima S. D'SOUZA. A rare muscular variation in the flexor compartment of the forearm International Journal of Anatomical Variations 2011; 4,115-116.

10. Binod Kumar Tamang, Pranoti Sinha, Rohit Kumar Sarda, Poonam Shilal, Murlimanju BV. Incidence and morphology of accessory head of flexor pollicis longus muscle - an anatomical study". Journal of Evolution of Medical and Dental Sciences 2013; 2, 36, 6800-6806.

\section{Source of Support: None Declared Conflict of Interest: None Declared}

Policy for Articles with Open Access

Authors who publish with MedPulse International Journal of Anatomy (Print ISSN: 2550-7621) (Online ISSN: 2636-4557) agree to the following terms: Authors retain copyright and grant the journal right of first publication with the work simultaneously licensed under a Creative Commons Attribution License that allows others to share the work with an acknowledgement of the work's authorship and initial publication in this journal.

Authors are permitted and encouraged to post links to their work online (e.g., in institutional repositories or on their website) prior to and during the submission process, as it can lead to productive exchanges, as well as earlier and greater citation of published work. 